CONNECTION BETWEEN OPTICAL OUTBURSTS AND SUPERLUMINAL MOTION IN THE MILLIARCSECOND RADIO STRUCTURE OF THE EXTRAGALACTIC OBJECTS

\author{
M.K. Babadzhanyants, E.T. Belokon' \\ Astronomical Observatory \\ Leningrad University \\ USSR
}

\title{
ABSTRACT
}

The analysis of the optical variability of 3C 345 along with the apparent superluminal motions in the milliarcsecond jet suggests a connection between these forms of activity.

The optical variability of the quasar 3C 345 is presented including our 18-year photographic observations and all published data by 1984 . The extrapolated moments of ejection of milliarcsecond jet components from nucleus coincide with the initial phases of the "slow" optical flares. The prediction about the arising of a new radiocomponent based on such treatment was confirmed later on. Some evidence of similar connection for the $\mathrm{N}$-galaxy $3 \mathrm{C}: 120$ is given.

It has been discovered in recent years that some extragalactic objects have apparent superluminal motions in their milliarcsecond jets. It is obvious that independently of the concrete physical interpretation of this phenomenon it represents an evidence of nuclear activity. Consequently this phenomenon may be connected with the variability of the optical continuum source. The object most suitable for studying such a relationship is the quasar $3 \mathrm{C} 345$. This superluminal object is the best-observed both by VLBI technique and by several optical monitoring programs which revealed very high degree of its optical variability (3.0 in the B-band) [1] .

\section{Optical data}

The quasar 3C 345 has been included on the optical monitoring program being performed at the Southern Station of the Astronomical Observatory of the Leningrad University since 1968.

Kinman et al. [2] were the first to analyse the optical variability of 3C 345. They had distinguished in 1965-1967 two variable components with different time-scales. We have analysed the optical variability of 3C 345 in the B-band using our own set of photographic observations obtained in 1968-1983 and the data of other authors published prior to 1984 (for references see [1]). The light curve of $3 C 345$ is shown in Fig. 1 (a).

The optical variations of $3 C 345$ can be subdivided into three variable components of approximately comparable amplitudes. Components 
I and II are shown by solid lines. Component I describes a systematic brightness increase in 1969-1983 of 0:05/yr. Component III has the timescale 10 days and includes besides the fluctuation with an amplitude 0!1-0!5 which are probably erratic, the peak-like fast (f) outbursts having the amplitude $\sim 1^{m}(B)$. The composite f-outburst light curve (Fig. 2) was formed by several such events observed in detail. F-outbursts show a remarkable constancy of amplitude expressed in magnitudes independently of the level of low-frequency components. Consequently there is proportionality of the f-outburst energy production and that of the underlying components. This property of the rapid fluctuations of $3 C 345$ was suspected 15 years ago by Penston and Cannon [3] and is supported now by subsequent observations.

The component II is the subject of our special attention. It is drawn as an envelope curve of the rapid fluctuations. This component may be considered as a number of slow (s) outbursts with the characteristic time-scale of $\sim 1-2$ years and amplitude of $\sim 1^{\text {m. }}$.

\section{VLBI observations and comparison with the optical outbursts}

Milliarcsecond structure of 3C 345 had been observed with VLBI technique systematically since 1971. It evidently shows superluminal motions of the compact jet components. Figure $1(b)$ shows the separation of the jet components from the core during the whole observing time interval at $\nu=\left(\begin{array}{ll}5 & 15\end{array}\right) \mathrm{GHz}$.

Since mid-1977 the component parameters of the milliarcsecond jet were determined using the hybrid mapping. It has clearly shown the existence of two compact jet components C2 and C3 (we use the designation of [4]) moving from the core with nearly equal and constant speeds. We have compared the linear extrapolation to zero separation of C2 and C3 with the optical variability [1]. The conclusion is that the epoch of zero separation of $\mathrm{C} 2$ and $\mathrm{C} 3$ found in such a manner are in good agreement with the beginning of the two s-outbursts that peaked in 1971 and 1976 (Fig. 1).

The early VLBI data (before 1977.5) were obtained using modelfitting techniques in which the milliarcsecond structure of 3C 345 was considered as a double source. A backward linear extrapolation of early data permitted to determine 1966.3 as the moment of the beginning of expansion. This epoch coincides wery well with the onset of the first s-outburst (Fig.1). Note that the component speeds differ more than twice.

We suppose that each optical s-outburst is accompanied by ejection or jet compact component. This assumption gives a new opportunity to interpret the observed changes of the milliarcsecond structure of $3 \mathrm{C}$ 345. Earlier a suggestion has been made which regards either the identifications of the jet component observed before 1977.5 with C2 or C3 or the bifurcation of the jet at mid-1977[5,6,7]. Such identifications require considerable acceleration of the early component or/and changes of its position angle.

The optical s-outburst of 1960-1967 gives an evidence of the probable existence of jet component C'1 ejected in 1966.3 different from $\mathrm{C} 2$ and $\mathrm{C} 3$. The presence of $\mathrm{C} 11, \mathrm{C} 2$ and $\mathrm{C} 3$ combined with their rapid flux density evolution (the half-life time 2.3 years [4]) can explain 
with sufficient confidence the whole complex picture of the milliarcsecond variations of $3 C 345$. For more detailed discussion of this question see [3].

On the base of the observed optical variability of $3 \mathrm{C} 345$ we have predicted [1] ejection of a new jei component associated with the last s-outburst of 1982-1983. But because of a gap in observations and insuificiency of the data onset of this s-outiurst (1981.0) is not so well deternined as in the case of preceding s-outbursts. Indeed, VLBI observations show the appearance of a new jet component $C 4[9,10]$ at the separation $\sim 0.3$ mass at 1931.1 . At this small separation C4 shows considerable acceleration and changes in its position angle. Therefore the linear extrapolation is impossible. However the fact of the appearance of $\mathrm{C} 4$ at this epoch undoubtedly associates this component with the last s-outburst. The values oi the proper motion and the position angle of $\mathrm{C} 4$ are approaching the mean values for the earlier components. If the speed of C4 found constant at separations $\geqq 1$ mas it will permit us to determine fornal (i.e. defined in such a manner as for earlier components using the same part of its trajectory at separations of $\sim(1-5)$ nas) epoch of zero separation for C4. Note also that the onset of the optical s-outbursts as found from the light curve do not necessarily correspond to a real epoch of its origin but rather to outbursts (for instance, if s-outbursis are overlapping).

A similar relation between the times of zero separation for the components $\mathrm{C} 2, \mathrm{C} 3, \mathrm{C} 4$ and the onsets of $3-\mathrm{mm}$ radio outbursts was found recently by Bregman et al. [11]. These radio outbursts have time-scales similar to those of the optical s-outbursts. At the same time large rapid variations at millimeter wavelengths similar to the optical component III are absent. Therefore it supposes the reality of our definition of optical s-outbursts.

The connection between the optical and VLBI variability is probably characteristic not only of $3 C$ 345 . Some evidence of the same phenomenon shows also the $\mathrm{N}$-galaxy $3 \mathrm{C} 120$. But because of insurificient optical data in this case !ve have applied another method. A mean irequency of the ejections of the jet components and frequency of the greatest maximum in the power spectrum of the optical variability were determined. The close agreement of the two values (303d and $305 \mathrm{~d}$ ) may be considered as an evidence of the existence of the sane type of connection as For $3 \mathrm{C}$ 345 .

In addition we want to call attention to such a feature: the majoricy of more than a dozen of superluminal objects For which observational data on their optical variability are available are known as OVV sources (the total range of optical variations may be as large as several magnitudes). Thus we conclude that the optical motions of the milliarcsecond jet components must have the same origin that supposes a comrion process which manifests itself over wide spectral range.

\section{REFERENCES}

1. M.K. Babadzhanyants, E.T. Belokon', Astrophysics, 21, 218, 1934.

2. T.D. Kinnan, E. Lamla, T. Ciurla, E. Harlan, C.A. Wirtanen, Ap.J., $152,352,1968$.

3. M.V. Penston, r.D. Cannon, Roy.Obs.Bull., 159, 85, 1970. 
4. S.C. Untrin, M.H. Cohen, T.J. Pearson, G.A. Seilstad, R.S. Simon, R.P. Linfield, R.C. Walker, Ap.J., 271, 535, 1983.

5. J. Schraml, I.I.K. Pauliny-Toth, A. Witzel, K.I. Kellernann, K.J. Johnston, J.H. Spenser, Ap.J., 251, L57, 1981.

6. M.H. Cohen, S.C. Univin, T.J. Pearson, G.A. Seielstad, R.S. Sinon, R.P. Linfield, r.C. Walker, Ap.J., 269, L1, 1983.

7. N. Bartel, T.A. Herring, M.I. Ratner, I.I. Shapiro, B.E. Corey, Nature, 319, 733, 1980.

8. M.K. Babadzhanyants, E.T. Belokon', Astrophysics, 23, 459, 1985.

9. J.A. Biretta, M.H. Cohen, S.C. Unwin, I.I.K. Paulini-Toth, Nature, $306,42,1983$.

10. R.L. Moore, A.S.C. Readhead, L. Baath, Nature, 306, 44, 1983.

11. J.N. Bregman, A.E. Glassgold, P.J. Huggins et al., Preprint NRAO-85/166, 1985. 


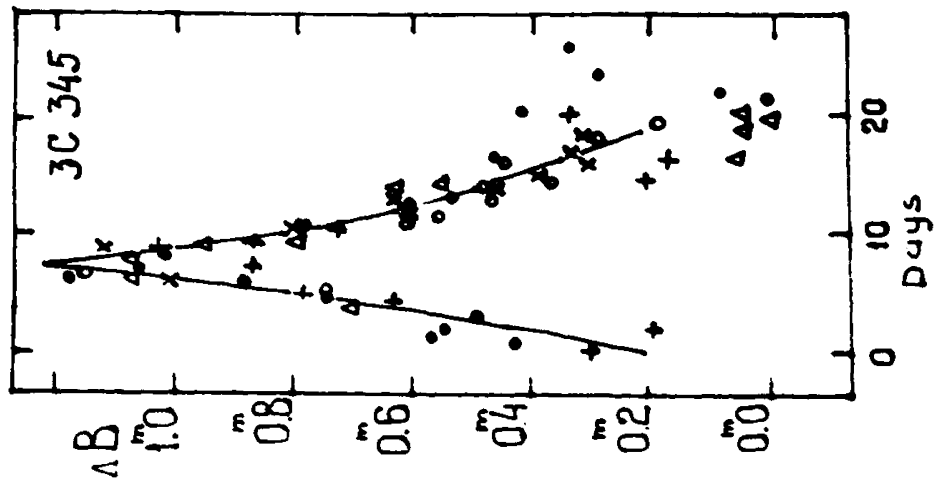

究

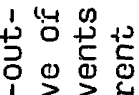
:-1 000

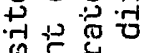

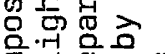

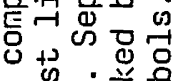

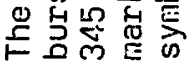

$\ddot{\sim}$

$\dot{\leftrightarrow}$

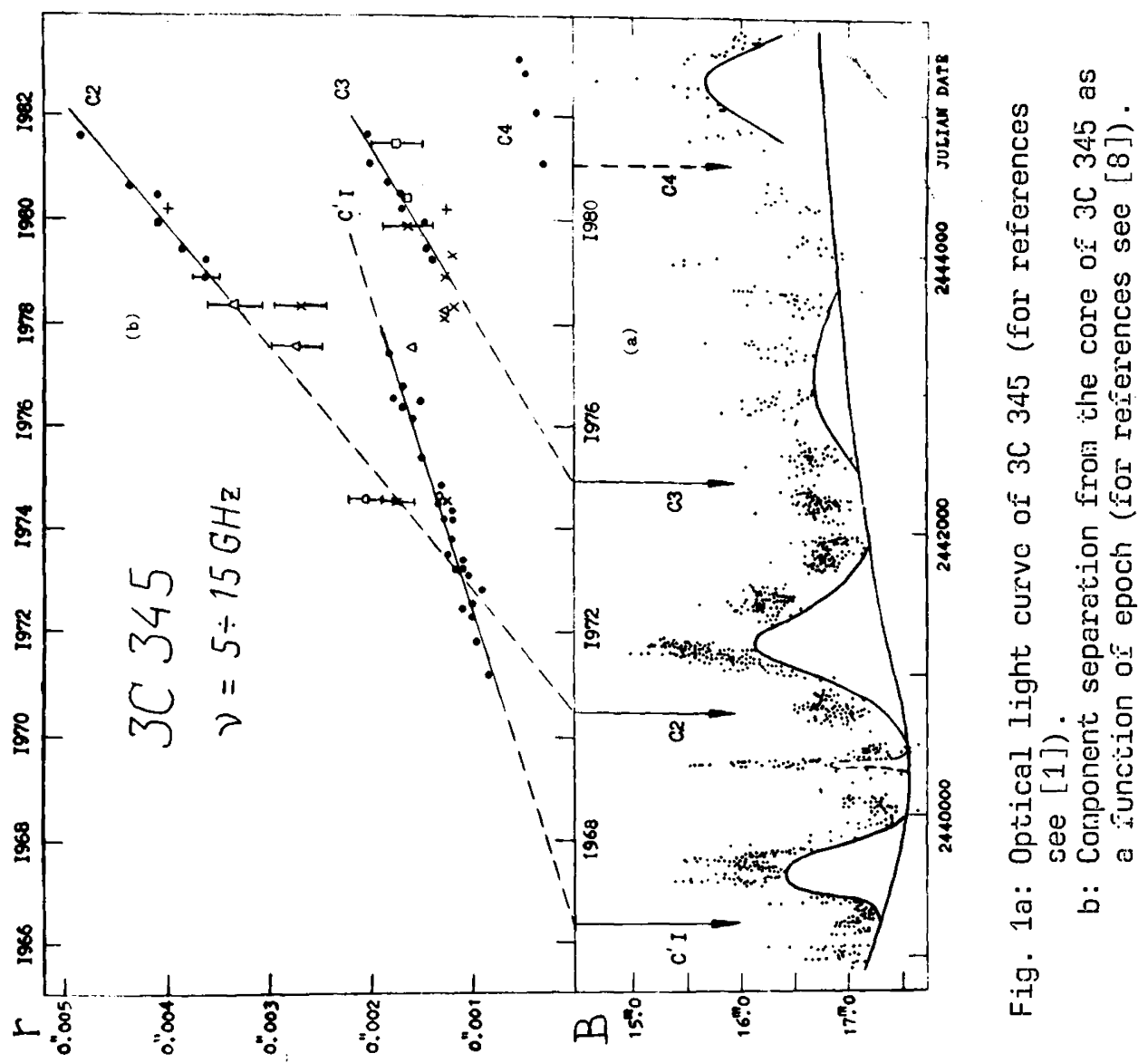

